\title{
Differential Expression of PKD2-Associated Genes in Autosomal Dominant Polycystic Kidney Disease
}

\author{
Yeon Joo Yook ${ }^{\dagger}$, Yu Mi $\mathrm{Woo}^{\dagger}$, Moon Hee \\ Yang, Je Yeong Ko, Bo Hye Kim, Eun Ji Lee, \\ Eun Sun Chang, Min Joo Lee, Sunyoung Lee \\ and Jong Hoon Park*
}

Department of Biological Science, Sookmyung Women's University, Seoul 140-742, Korea

\begin{abstract}
Autosomal dominant polycystic kidney disease (ADPKD) is characterized by formation of multiple fluid-filled cysts that expand over time and destroy renal architecture. The proteins encoded by the PKD1 and PKD2 genes, mutations in which account for nearly all cases of ADPKD, may help guard against cystogenesis. Previously developed mouse models of PKD1 and PKD2 demonstrated an embryonic lethal phenotype and massive cyst formation in the kidney, indicating that PKD1 and PKD2 probably play important roles during normal renal tubular development. However, their precise role in development and the cellular mechanisms of cyst formation induced by PKD1 and PKD2 mutations are not fully understood. To address this question, we presently created Pkd2 knockout and PKD2 transgenic mouse embryo fibroblasts. We used a mouse oligonucleotide microarray to identify messenger RNAs whose expression was altered by the overexpression of the PKD2 or knockout of the $P k d 2$. The majority of identified mutations was involved in critical biological processes, such as metabolism, transcription, cell adhesion, cell cycle, and signal transduction. Herein, we confirmed differential expressions of several genes including aquaporin-1, according to different PKD2 expression levels in ADPKD mouse models, through microarray analysis. These data may be helpful in PKD2-related mechanisms of ADPKD pathogenesis.
\end{abstract}

Keywords: cystogenesis, MEF cells, microarray, PKD2, polycystic kidney disease

\footnotetext{
†These authors contributed equally to this work. ${ }^{*}$ Corresponding author: E-mail parkjh@sookmyung.ac.kr Tel +82-2-710-9414, Fax +82-2-2077-7322

Received 30 January 2012, Revised 15 February 2012, Accepted 16 February 2012
}

\section{Introduction}

Autosomal dominant polycystic kidney disease (ADPKD) is a common monogenetic disorder, affecting as many as 1 in 500 people in the general population. The disease is characterized by the formation of multiple fluid-filled renal cysts that expand over time and destroy the architecture of the kidney. Five percent of all cases of chronic renal failure are due to ADPKD, and approximately $50 \%$ of ADPKD patients will develop end-stage renal disease by the time they are 60 years of age [1].

In the early stages of ADPKD, numerous cysts begin to enlarge from many segments of the kidney. Later, the enlarged regions disassociate from the original nephron to form the actual cysts, which continue to enlarge due to proliferation of epithelial cells and fluid secretion into the cyst lumen. Progressive renal cyst formation and enlargement result in a loss of renal function and hypertension and culminate in renal failure. It is generally believed that cysts enlarge by means of abnormal cell growth, forming what is essentially a fluid-filled tumor that fills by transepithelial fluid secretion. Cyst enlargement may then lead to extracellular matrix remodeling, interstitial fibrosis, a general inflammatory response, and disruption of the normal renal parenchyma, which then interfere with glomerular filtration and vascular blood flow, giving rise to cell death by apoptosis and ultimately renal failure [2].

Many studies have indicated that most cases of ADPKD are accounted for by mutations in the PKD1 and $P K D 2$ genes, encoding the transmembrane protein polycystin-1 (PC1) and polycystin-2 (PC2), respectively. Mice with targeted mutations in the PKD1 or PKD2 genes develop cystic kidneys during embryogenesis, and $A D P K D$ in humans is associated with mutations in the PKD1 or PKD2 genes $[3,4]$. In addition, the product of the $P K D 1$ gene, $P C 1$, has been implicated in a variety of pathways tied to proliferation, including G-protein signaling and the Wnt, activator protein 1 (AP-1), and Janus kinase-signal transducers and activators of transcription cascades [5, 6]. Moreover, depletion of PC1 has been shown to increase cell growth, whereas its overexpression slows cell growth, indicating that PC1 may negatively regulate cell proliferation $[7,8]$. $P C 2$, the protein product of $P K D 2$, has also been implicated in cell cycle regulation via its calcium channel activity and stimulation of AP-1 [9, 10]; however, there has been little direct evidence tying PC2 to this process. 
Here, we created a number of related cell lines that varied in their expression of PC2. We describe studies designed to identify target genes under the control of the PKD2 using Pkd2 knockout (KO) and PKD2 transgenic (TG) mouse embryo fibroblasts (MEFs). We used a mouse $30 \mathrm{~K}$ whole gene oligonucleotide microarray to identify messenger RNAs whose expression was altered by the overexpression of the PKD2 or KO of the Pkd2 in MEF cells.

\section{Methods}

\section{Establishment of MEF derived from wild-type and PKD2 mutation embryos}

MEF were acquired from PKD2 TG embryos or $P k d 2 \mathrm{KO}$ embryos during development (13.5 days) [11]. Heads and limes were removed from embryos. The remaining embryonic tissues were minced and dispersed in $0.05 \%$ trypsin prior to incubation at $37^{\circ} \mathrm{C}$ for $15 \mathrm{~min}$. Cells were plated in Dulbecco's Modified Eagle's Medium of Defined Minimal Essential medium (Welgene Biotech, Taipei, Taiwan) supplemented with $10 \%$ fetal bovine serum (Welgene Biotech) and were cultured at $37^{\circ} \mathrm{C}$ in an atmosphere of $5 \% \quad \mathrm{CO}_{2}$ until confluent growth was achieved. MEF cells were frozen as stocks at the second passage and were used for the subsequent studies at the third passage.

\section{Microrray hybridization and data analysis}

Total RNA was prepared using a commercial kit (Qiagen, Valancia, CA, USA) according to the manufacturer's instructions. Gene expression profiles were obtained using the CodeLink Uniset Mouse Expression Bioarray (Amersham, Buckinghamshire, UK). The 30,000 gene probes contained in the bioarray allows the detection of differences in gene expression as low as 1.3-fold with $95 \%$ confidence. Ten micrograms of total RNA was amplified, and labeled cDNA was produced. Biotin-labeled cDNA was hybridized to the array overnight in a shaking incubator at $37^{\circ} \mathrm{C}$, and excess target sequences were washed away using a series of saline sodium citrate washes. The array was stained by treatment with streptavidin-Alexaflour 647 (Molecular Probes, Eugene, OR, USA), the excess was washed away, and the array was scanned at an excitation wavelength of $632 \mathrm{~nm}$ using a GenePix scanner (Molecular Devices, Sunnyvale, CA, USA). The resulting image was quantified, and the intensity of each spot was divided by the median spot intensity to provide a scaled and comparable number across multiple arrays.

\section{DNA microarray scanning and analysis}

Microarrays were scanned using an Arraywox scanner (Applied Precision, Seattle, WA, USA), analyzed using ImaGene version 5.1 software (Biodiscovery, Segundo, CA, USA), and normalized using Genesight version 3.2 software (Biodiscovery). Normalization was performed by subtracting the means of all genes. The data normalized by Genesight were compared using an M/A plot. Genes differentially expressed were identified by intensity differences, after subtracting the background intensity. Genes showing expression changes of at least 2-fold were selected, and these selected genes were clustered by the hierarchical method.

\section{Clustering algorithm}

The normalized log ratio corresponding to each time point was exported to the software for clustering algorithm. Acuity version 3.1 (Molecular Devices) was used for the 'gene shaving' algorithm. To estimate the number of clusters in a dataset, the 'Gap Statistics' attribute of the Acuity software package was used. The cluster number, estimated by gap statistics, was used for an input parameter in gene shaving. After gene shaving, a single linkage was used for hierarchical clustering.

\section{Semi-quantitative and real-time RT-PCR}

Total RNA was prepared using a commercial kit (Qiagen) according to the manufacturer's instructions. Singlestrand cDNA was synthesized by incubating $5 \mu \mathrm{g}$ total RNA with 200 units AMV, $100 \mathrm{nM}$ oligo(dT)12-18, $1 \mathrm{mM}$ dNTP mixture, and 40 units RNase inhibitor at $42^{\circ} \mathrm{C}$ for $1 \mathrm{~h}$ in a final volume of $25 \mu \mathrm{L}$. The reaction was terminated by incubation at $70^{\circ} \mathrm{C}$ for $15 \mathrm{~min}$. The initial amount of mRNA and reaction conditions were optimized to obtain linearity for mouse $18 s r R N A$. For semi-quantitative reverse transcription (RT)-PCR, the used primers were: human $P K D 2$, forward 5'-CGTGCCCCAGCCCAGTC3 ' and reverse 5'-TTCCAGTACAGCCCATCCAATAAG-3'; mouse Pkd2, forward 5'-TGCGAGGGCTGCGAGGTC-3' and reverse 5'- TGTCAGCTTGCGTGTGGTTGC-3'; mouse 18s rRNA, forward 5'-GTAACCCGTTGCACCCCATT-3' and reverse 5'-CCATCCAATCGGTAGTAGCG-3'. RT-PCR cycling conditions were as follows: $10 \mathrm{~min}$ at $95^{\circ} \mathrm{C}, 25$ cycles of $50 \mathrm{~s}$ at $94^{\circ} \mathrm{C}, 50 \mathrm{~s}$ at $57^{\circ} \mathrm{C}, 50 \mathrm{~s}$ at $72^{\circ} \mathrm{C}$, and $5 \mathrm{~min}$ at $72^{\circ} \mathrm{C}$. The amplified products were separated on a $1 \%$ agarose gel. For real-time PCR, the used primers were: mouse $\beta$-actin, (forward 5'-GACGATGCTCC CCGGGCTGTATTC-3' and reverse 5'-TCTCTTGCTCTG GGCCTCGTCACC-3') used as a positive control; mouse peroxisome proliferator-activated receptor $\gamma(P P A R-\gamma)$, 
forward 5'-TCTTAACTGCCGGATCCACAAAAA-3' and reverse 5'-ATCTCCGCCAACAGCTTCTCCTTC-3'; mouse protein kinase $\alpha(P K C \alpha)$, forward 5'- GGGCAGCCTCC GTTTGATGGT-3' and reverse 5'-CGCTTGGCAGGGTGT TTGGTC-3'; mouse Wnt4, forward 5'-GCCATCGAGGAG TGCCAATACC-3' and reverse 5'-GGCCACACCTGCTGA AGAGATG-3'; mouse integrin $\alpha 4$ (/TG $\alpha 4$ ), forward 5'GTAGCCCCAGTGGAGAGCCTTGTG-3' and reverse 5'ATGCCAGTGGGGAGTTTGTTATCG-3'; mouse IL6ST, forward 5'-TGAATCGGACCCACTTGAGAGG-3' and reverse 5'-CAGGAGCGGCTTGTTTGAGGTA-3'; mouse aquaporin 1 (AQP1), forward 5'-GGAGGCGCCGAGACTTAGGT-3' and reverse 5'-GCGGGTGAGCACAGCAGAGC-3'; mouse transforming growth factor- $\beta 2$ (TGF- $\beta 2$ ), forward 5'TCATCCCGAATAAAAGCGAAGAGC-3' and reverse 5'AGGGCAACAACATTAGCAGGAGAT-3'. Real-time RT-PCR was performed using the real-time SensiMixPlus SYBR kit as described by the manufacturer's instructions (Quantance, London, UK).

\section{Western blot analysis}

Proteins were extracted using radio-immunoprecipitation assay buffer in MEF cells. Proteins were separated by $12 \%$ sodium dodecyl sulfate-polyacryamide gel electrophoresis and were transferred to a polyvinylidene fluoride membrane (Millipore, Bellerica, MA, USA). Membranes were blocked with $5 \%$ non-fat dry milk and were incubated with various antibodies. Antibodies to PC2 (anti-human and anti-mouse) and AQP1 were obtained from Santa Cruz Biotechnology (Santa Cruz, CA, USA). Antibodies to $\beta$-actin were obtained from Sigma-Aldrich (St. Louis, MO, USA). Anti-Wnt4 was obtained from R\&D Systems (Minneapolis, MN, USA). Each membrane was washed with phosphate-buffered saline-Tween, and immunocomplexes were detected by enhanced chemiluminescence (Amersham).

\section{Results}

\section{Confirmation of PKD2 expression in Pkd2 KO and PKD2 TG MEFs}

Mouse $P k d 2$ mRNA was revealed in KO wild-type (KOWT) MEF cells but not in KO MEF cells. Also, Pkd2 mRNA was expressed in TG wild-type (TGWT) MEF cells and PKD2 TG MEF cells. Human PKD2 mRNA was only expressed in PKD2 TG MEF cells obtained from PKD2 TG embryos following injection of the human PKD2 transgene (Fig. 1A). Consistently, the expression level of polycystin-2 was lower in KO MEFs than KOWT MEFs as well as higher in TG MEFs than TGWT MEFs (Fig. 1B).

\section{Microarray gene expression analysis}

We used a mouse $30 \mathrm{~K}$ whole gene oligonucleotide microarray to identify mRNAs whose expression was altered by the Pkd2 KO and PKD2 TG MEF cells. The normalized log ratios corresponding to each time point were exported to the software for clustering algorithm. We used Axon's Acuity 3.1 (Axon Instruments, Union City, CA, USA) for using the 'gene shaving' algorithm. To estimate the number of clusters in a dataset, we used 'Gap Statistics' in Acuity 3.1. The cluster number estimated by gap statistics was used for an input parameter in gene shaving. After gene shaving, we used a single linkage hierarchical clustering. The single linkage hierarchical clustering method divides 8 'gene shaving' clusters (Fig. 2). The majority of genes whose expression was appreciably altered encoded proteins involved in critical biological processes, such as metabolism, transcription, cell adhesion, cell cycle, and signal transcription. Forty- five genes whose expression was changed by 2 -fold or greater were identified (Table 1).

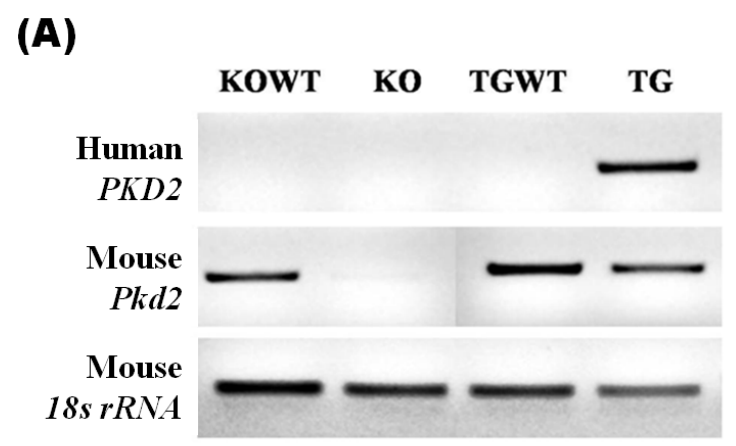

(B)

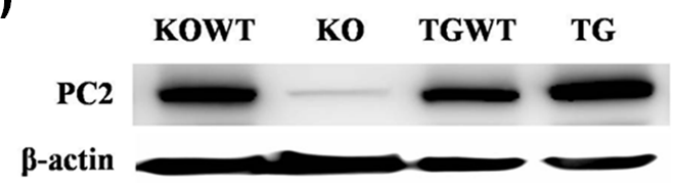

Fig. 1. Establishment of $P k d 2 \mathrm{KO}$ and PKD2 TG MEFs. (A) Semi-quantitative reverse transcription-PCR analysis of $P k d 2$ gene in $P k d 2 \mathrm{KO}$ and PKD2 TG MEF cells. Humanor mouse-specific PKD2 primer sets were used. 18s rRNA was used as a loading control. (B) Western blot analysis of polycystin 2 (PC2) expression in Pkd2 KO and PKD2 TG MEF cells. MEFs, mouse embryo fibroblasts; KO, knockout (MEF); TG, transgenic (MEF); KOWT, knockout wild-type (MEF); TGWT, transgenic wild-type (MEF). 
(A)

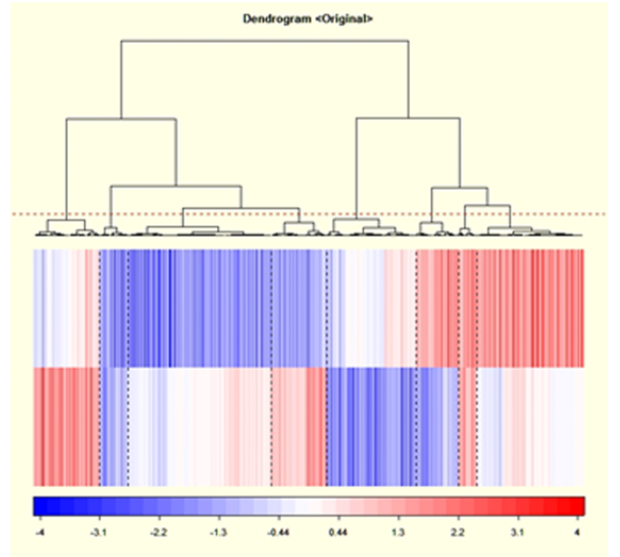

(B)

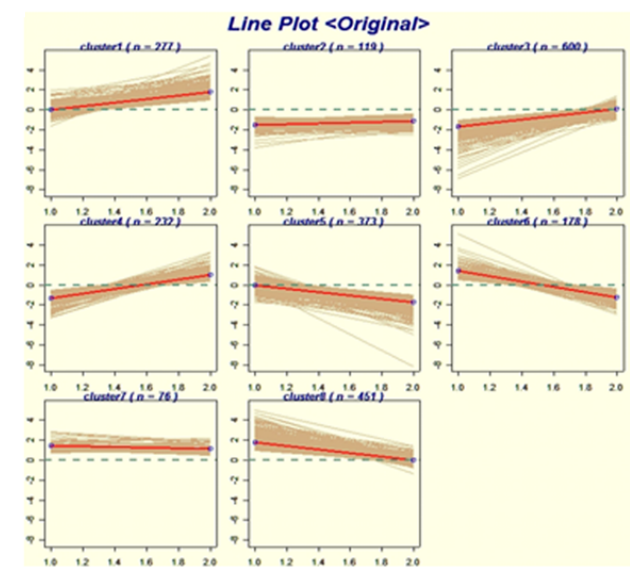

Fig. 2. Clustering of gene expression patterns in $P k d 2$ mutant MEF cell lines. We performed clustering analysis microarray data between KO set ratio data and TG set ratio data using the graphical user interface (GUI) system. (A) Gene expression profiles of 2,306 probe sets distributed in a dendrogram. (B) Line plots of each cluster. KO set, ratio between KO MEF and KOWT MEF; TG set, ratio between TG MEF and TGWT MEF. MEFs, mouse embryo fibroblasts; KO, knockout (MEF); TG, transgenic (MEF); KOWT, knockout wild-type (MEF); TGWT, transgenic wild-type (MEF).

\section{Verification of candidate genes by quantitative RT-PCR and immunoblot analysis}

To verify the results obtained by cDNA microarray analysis, expression patterns for selected genes were confirmed at the RNA and protein levels. We selected seven genes among the 45 genes (Fig. $3 A$ ). As result of realtime RT-PCR, the expression patterns of $P K C \alpha, I T G \alpha 4$, and $A Q P 1$ were identical to microarray analysis in $P k d 2$ KO MEF cells (Fig. 3B). In contrast, 6 genes, except for TGF- $\beta 2$, were identically expressed in PKD2 TG MEF cells (Fig. $3 C$ ). Especially, the $A Q P 1$ gene may be significantly regulated by differential $P k d 2$ gene expression level. Furthermore, to confirm the AQP1 protein expression level, western blot analysis was performed using MEF cells as well as kidney tissues obtained from Pkd2 KO (or heterozygote) and PKD2 TG mice. As result, AQP1 protein level was consistent with mRNA levels in both MEFs and tissues (Fig. 4).

\section{Discussion}

Although remarkable progress toward understanding the genetics and pathophysiology of ADPKD has been made to date, it is still unclear how mutations in disease-causing genes trigger cystogenesis and what important role other molecules play in the cystic phenotype [12]. Our aim here was to identify genes associated with the cystic phenotype using Pkd2 KO and PKD2 TG MEF cells. In a previous study, overexpression of human PKD2 led to anomalies in tubular function, due to abnormalities in tubule morphogenesis [13]. Similar findings have been made for the PKD1 gene. For this gene, gain/loss of function and haploinsufficiency leads to cystogenesis, with the severity of the phenotype being related to the level of imbalance. More severe phenotypes are seen for gain and loss of function than for haploinsufficiency [4]. In the present study, the association between cystogenesis and the loss or gain of PKD2 supports the idea that PC2 functions through Pkd2 mutant MEF cells.

Presently, we found Wnt4 to be up-regulated in Pkd2 KO MEF cells and down-regulated in PKD2 TG MEF cells. Wnt4, a member of the Wnt family of lipid-modified secreted glycoproteins, plays central roles in the initial stages of the tubulogenic program. Wnt4 is expressed in the pretubular aggregate, as one of the first molecular responses to inductive signaling mediated by ureteric epithelium [14].

AQP1 was observed to be up-regulated in both $P k d 2$ KO and PKD2 TG MEF cells. AQP1 is a channel-forming integral membrane protein found in erythrocytes and water-transporting tissues, including the kidney, where it is localized to the proximal tubules and thin descending limbs of Henle's loop. The selective expression pattern of AQP1 in human nephrogenesis suggests that proximal tubules and thin descending limbs become permeable to water early in development. A predominantly apical expression of AQP1 is found during a critical period of the nephrogenesis, during formation of the proximal tubule, as well as in the early stage of ADPKD; in normal tubules, distribution is both apical and basolateral [15]. Another interesting defect in urine-concen- 
trating ability was described in the Aqp1 knockout mouse. Although these mice were grossly normal in terms of survival and appearance, they were vulnerable to water deprivation and became severely dehydrated. These results suggest that AQP1 is required for the formation of concentrated urine [16].

We have found PKC- $\alpha$ and $\mathrm{Bcl}-2$ associated transcription factor to be down-regulated in Pkd2 KO MEF cells and up-regulated in PKD2 TG MEF cells. Recent observations in animal models of PKD have implicated apoptosis in its pathogenesis. PKC- and Erk-dependent pathways are critical components of the cell survival by sup- pressing the apoptosis [17]. We also have demonstrated the role for $P K D 2$ in cellular adhesion processes. In this study, ITG $\alpha 4$ was up-regulated in Pkd2 KO MEF cells and down-regulated in PKD2 TG MEF cells. It suggests that adhesion molecule such as ITG $\alpha 4$ may be associated with PKD2 mutation-related pathogenesis.

In conclusion, by using the cDNA microarray technique, we have found several candidate genes that may be involved in cystogenesis: PKC $\alpha$, ITG $\alpha 4$ and AQP1. These genes were affected by $P K D 2$ expression and may be related to cyst formation of kidney together with the PKD2 gene.

Table 1. Genes deregualted by differential $P k d 2$ expression

\begin{tabular}{|c|c|c|c|}
\hline \multirow{2}{*}{$\begin{array}{c}\text { GenBank } \\
\text { Accession no. }\end{array}$} & \multirow{2}{*}{ Definition } & \multicolumn{2}{|c|}{ Fold change } \\
\hline & & KO & TG \\
\hline \multicolumn{4}{|l|}{ Proliferation } \\
\hline NM_008275.2 & Homeo box D13 & 1.00 & 0.38 \\
\hline NM_008783.1 & Pre B-cell leukemia transcription factor 1 & 0.38 & 1.93 \\
\hline NM_011245.2 & RAS protein-specific guanine nucleotide releasing factor 1 & 3.64 & 1.36 \\
\hline NM_009367.1 & Transforming growth factor, beta 2 & 0.49 & 1.22 \\
\hline NM_013693.1 & Tumor necrosis factor & 0.30 & 0.27 \\
\hline \multicolumn{4}{|l|}{ Apoptosis } \\
\hline NM_001025393.1 & BCL2-accosiated transcription factor 1 & 0.36 & 1.23 \\
\hline NM_007465.1 & Baculoviral IAP repeat-containing 2 & 1.32 & 0.20 \\
\hline NM_001038658.1 & FAS apoptotic inhibitory molecule 2 & 0.81 & 0.44 \\
\hline NM_011563.2 & Peroxiredoxin 2 & 0.48 & 0.85 \\
\hline NM_011101.1 & Protein kinas $\mathrm{C}$, alpha & 0.21 & 1.49 \\
\hline NM_011609.2 & Tumor necrosis factor receptor superfamily, member 1a & 0.24 & 0.93 \\
\hline \multicolumn{4}{|l|}{ PPAR gamma } \\
\hline NM_011146.1 & Peroxisome proliferator activated receptor gamma & 0.37 & 1.64 \\
\hline NM_008904.1 & Peroxisome proliferative activated receptor, gamma, coactivator 1 alpha & 0.59 & 0.49 \\
\hline NM_133249.2 & Peroxisome proliferative activated receptor, gamma, coactivator 1 beta & 0.20 & 0.37 \\
\hline \multicolumn{4}{|l|}{ Wnt } \\
\hline NM_011718.1 & Wingless related MMTV integration site $10 \mathrm{~b}$ & 0.31 & 0.90 \\
\hline NM_008058.1 & Frizzled homolog 8 (drosophila) & 0.43 & 1.09 \\
\hline NM_009523.1 & Wingless related MMTV integration site 4 & 5.21 & 0.72 \\
\hline NM_009331.2 & Transcription factor, T-cell specific & 2.99 & 0.74 \\
\hline \multicolumn{4}{|l|}{ Chemokine } \\
\hline NM_008176.1 & Chemokine ( $\mathrm{C}-\mathrm{X}-\mathrm{C}$ motif) ligand 1 & 3.97 & 1.08 \\
\hline NM_021704.2 & Chemokine ( $\mathrm{C}-\mathrm{X}-\mathrm{C}$ motif) ligand 12 & 0.44 & 1.05 \\
\hline NM_009911.2 & Chemokine (C-X-C motif) receptor 4 & 2.66 & 0.55 \\
\hline NM_009140.1 & Chemokine ( $\mathrm{C}-\mathrm{X}-\mathrm{C}$ motif) ligand 2 & 2.75 & 1.27 \\
\hline \multicolumn{4}{|l|}{ Aquaporin } \\
\hline NM_007472.1 & Aquaporin 1 & 1.43 & 2.27 \\
\hline NM_016689.2 & Aquaporin 3 & 1.23 & 2.01 \\
\hline \multicolumn{4}{|l|}{ Interleukin } \\
\hline NM_010560.2 & Interleukin 6 signal transducer & 0.31 & 1.61 \\
\hline NM_008353.1 & Interleukin 12 receptor, beta1 & 2.20 & 1.89 \\
\hline NM_008356.1 & Interleukin 13 receptor, alpha2 & 4.03 & 0.85 \\
\hline NM_134437.1 & Interleukin 17 receptor D & 2.28 & 1.06 \\
\hline NM_133193.1 & Interleukin 1 receptor-like 2 & 2.41 & 0.85 \\
\hline \multicolumn{4}{|l|}{ Integrin } \\
\hline NM_010576.3 & Integrin alpha 4 & 2.55 & 0.48 \\
\hline
\end{tabular}

$\mathrm{KO}$, knockout; TG, transgenic. 
(A)
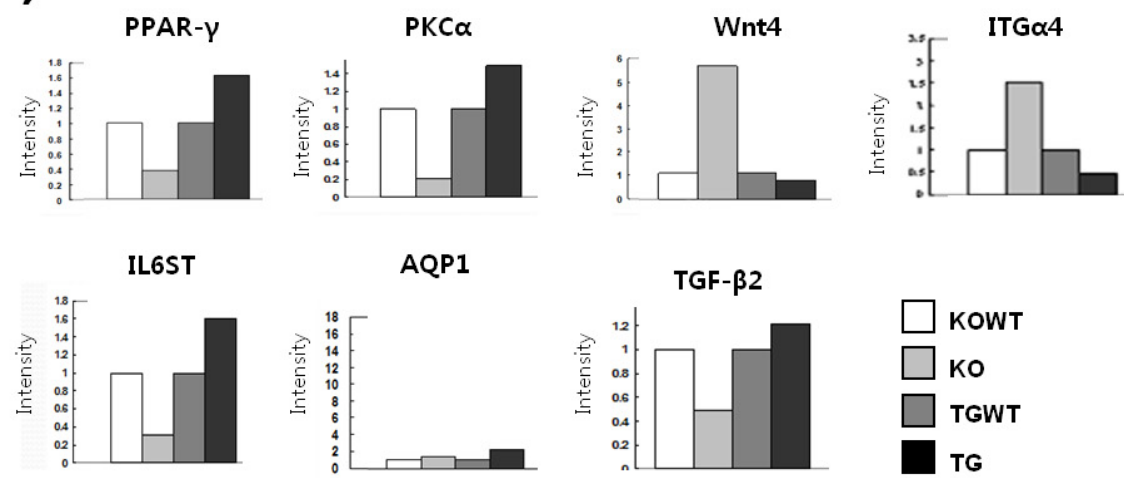

AQP1
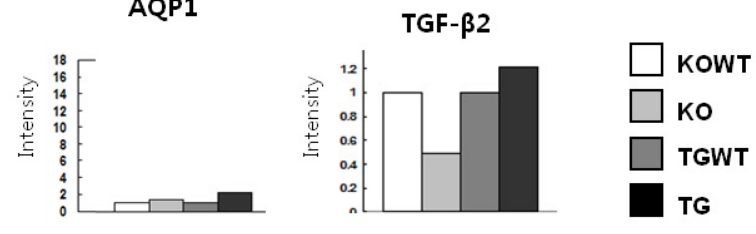

(B)

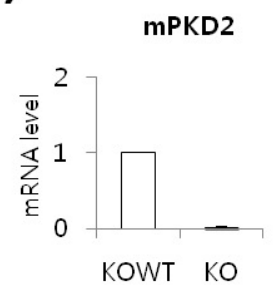

PKC $\alpha$

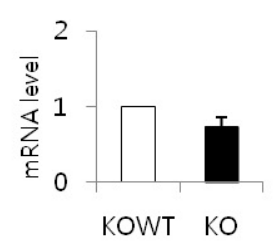

ITG $\alpha 4$

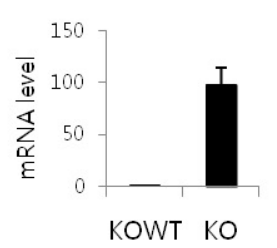

AQP1

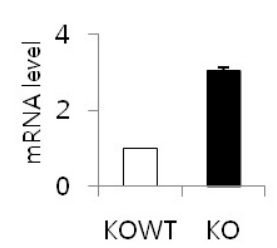

PPARY

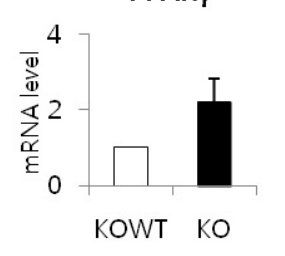

Wnt4

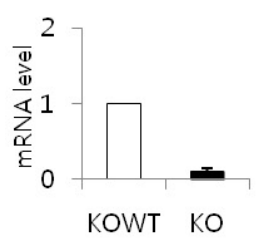

IL6ST

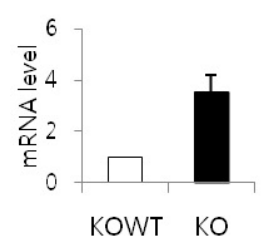

TGF $\beta 2$

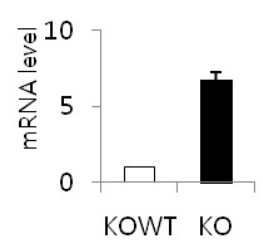

(C)

hPKD2

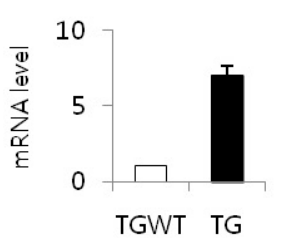

PKC $\alpha$

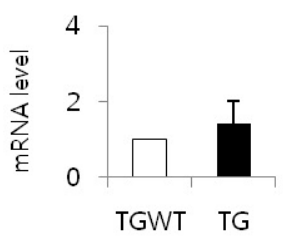

ITG $\alpha 4$

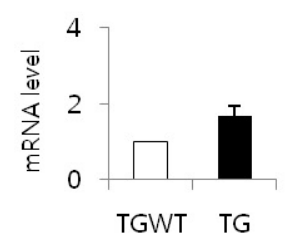

AQP1

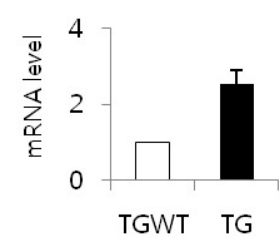

PPAR $-\gamma$

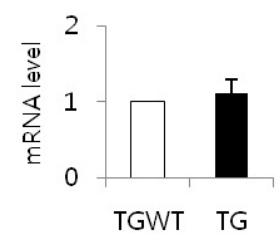

Wnt4

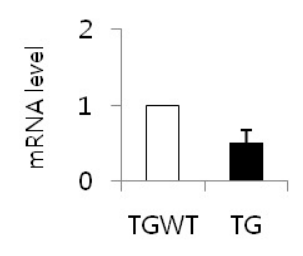

IL6ST

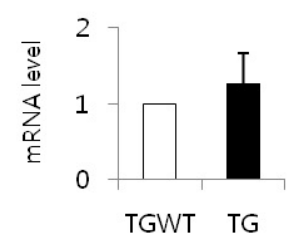

TGF- $\beta 2$

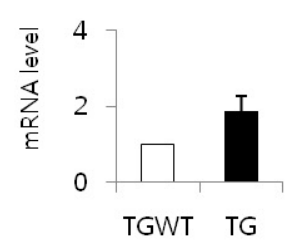

Fig. 3. Expression verification of candidate genes at the mRNA level. (A) Microarray data for selective genes including $P P A R \gamma$, $P K C \alpha$, Wnt4, ITG $\alpha$ 4, IL6ST, AQP1, and TGF- $\beta 2$. (B, C) Real-time reverse transcription-PCR analysis of gene expression for selective genes in $P k d 2 \mathrm{KO}$ and $P K D 2$ TG MEF cells. $\beta$-actin primer was used as the internal control. The experiment was performed in triplicate. PPAR- $\gamma$, peroxisome proliferator-activated receptor $\gamma$; PKC $\alpha$, protein kinase $\alpha$; ITG $\alpha 4$, integrin $\alpha 4$; $A Q P 1$, aquaporin 1; TGF- $\beta 2$, transforming growth factor- $\beta 2$; KO, knockout; TG, transgenic; MEF, mouse embryo fibroblasts; KOWT, knockout wild-type (MEF); TGWT, transgenic wild-type (MEF). 
(A)

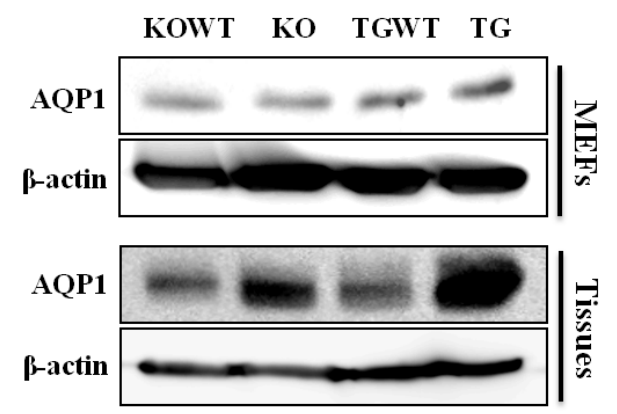

(B)

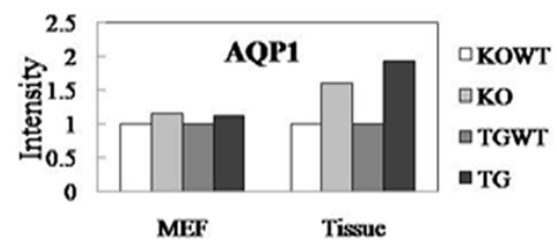

Fig. 4. Expression verification of $A Q P 1$ at the protein level. (A) Western blot analysis of AQP1 in MEFs and kidney tissues derived from $P k d 2 \mathrm{KO}$ (or heterozygote) and PKD2 TG mice. PKD2 TG and TGWT mice: $18 \mathrm{mo}$; Pkd2 heterozygote and KOWT mice: $12 \mathrm{mo}$. Anti- $\beta$-actin was used as the internal control. (B) For quantification of western blot, Multigauge software (Fuji Film) was used. AQP1, aquaporin 1; MEF, mouse embryo fibroblasts; KO, knockout; TG, transgenic; KOWT, knockout wild-type (MEF); TGWT, transgenic wild-type (MEF).

\section{Acknowledgments}

This work was supported by a Sookmyung Women's University Research Grant (2010).

\section{References}

1. Grimm DH, Karihaloo A, Cai Y, Somlo S, Cantley LG, Caplan MJ. Polycystin-2 regulates proliferation and branching morphogenesis in kidney epithelial cells. J Biol Chem 2006;281:137-144.

2. Wilson PD, Falkenstein D. The pathology of human renal cystic disease. Curr Top Pathol 1995;88:1-50.

3. Kim K, Drummond I, Ibraghimov-Beskrovnaya O, Klinger $\mathrm{K}$, Arnaout MA. Polycystin 1 is required for the structural integrity of blood vessels. Proc Natl Acad Sci USA 2000;97:1731-1736.

4. Wu G, D'Agati V, Cai Y, Markowitz G, Park JH, Reynolds
$\mathrm{DM}$, et al. Somatic inactivation of Pkd2 results in polycystic kidney disease. Cell 1998;93:177-188.

5. Arnould T, Kim E, Tsiokas L, Jochimsen F, Grüning W, Chang JD, et al. The polycystic kidney disease 1 gene product mediates protein kinase $\mathrm{C}$ alpha-dependent and C-Jun N-terminal kinase-dependent activation of the transcription factor AP-1. J Biol Chem 1998;273: 6013-6018.

6. Parnell SC, Magenheimer BS, Maser RL, Zien CA, Frischauf AM, Calvet JP. Polycystin-1 activation of c-Jun $\mathrm{N}$-terminal kinase and AP-1 is mediated by heterotrimeric G proteins. J Biol Chem 2002;277:19566-19572.

7. Boletta A, Qian F, Onuchic LF, Bhunia AK, Phakdeekitcharoen $\mathrm{B}$, Hanaoka $\mathrm{K}$, et al. Polycystin-1, the gene product of PKD1, induces resistance to apoptosis and spontaneous tubulogenesis in MDCK cells. Mol Cell 2000;6:1267-1273.

8. Kim H, Bae Y, Jeong W, Ahn C, Kang S. Depletion of PKD1 by an antisense oligodeoxynucleotide induces premature G1/S-phase transition. Eur J Hum Genet 2004; 12:433-440.

9. Gallagher AR, Cedzich A, Gretz N, Somlo S, Witzgall R. The polycystic kidney disease protein PKD2 interacts with $\mathrm{Hax}-1$, a protein associated with the actin cytoskeleton. Proc Natl Acad Sci U S A 2000;97:4017-4022.

10. Luo Y, Vassilev PM, Li X, Kawanabe Y, Zhou J. Native polycystin 2 functions as a plasma membrane $\mathrm{Ca} 2+-$ permeable cation channel in renal epithelia. Mol Cell Biol 2003;23:2600-2607.

11. Park EY, Sung YH, Yang MH, Noh JY, Park SY, Lee $\mathrm{TY}$, et al. Cyst formation in kidney via B-Raf signaling in the PKD2 transgenic mice. J Biol Chem 2009;284: 7214-7222.

12. Husson $H$, Manavalan $P$, Akmaev VR, Russo RJ, Cook $B$, Richards $B$, et al. New insights into ADPKD molecular pathways using combination of SAGE and microarray technologies. Genomics 2004;84:497-510.

13. Burtey S, Riera M, Ribe E, Pennekamp P, Passage E, Rance $\mathrm{R}$, et al. Overexpression of PKD2 in the mouse is associated with renal tubulopathy. Nephrol Dial Transplant 2008;23:1157-1165.

14. Park JS, Valerius MT, McMahon AP. Wnt/beta-catenin signaling regulates nephron induction during mouse kidney development. Development 2007;134:2533-2539.

15. Devuyst O, Burrow CR, Smith BL, Agre P, Knepper MA, Wilson PD. Expression of aquaporins-1 and -2 during nephrogenesis and in autosomal dominant polycystic kidney disease. Am J Physiol 1996;271(1 Pt 2):F169-F183.

16. Yamamoto T, Sasaki S. Aquaporins in the kidney: emerging new aspects. Kidney Int 1998;54:1041-1051.

17. Banzi M, Aguiari G, Trimi V, Mangolini A, Pinton P, Witzgall $\mathrm{R}$, et al. Polycystin-1 promotes PKCalphamediated NF-kappaB activation in kidney cells. Biochem Biophys Res Commun 2006;350:257-262. 